\title{
Oxidative Stress in Alzheimer's and Parkinson's Diseases: Insights from the Yeast Saccharomyces cerevisiae
}

\author{
Catarina Pimentel, Liliana Batista-Nascimento, \\ Claudina Rodrigues-Pousada, and Regina A. Menezes \\ Instituto de Tecnologia Química e Biológica, Universidade Nova de Lisboa, Oeiras, Portugal \\ Correspondence should be addressed to Regina A. Menezes, rmenezes@itqb.unl.pt
}

Received 12 February 2012; Revised 3 April 2012; Accepted 3 April 2012

Academic Editor: Marcos Dias Pereira

Copyright (C) 2012 Catarina Pimentel et al. This is an open access article distributed under the Creative Commons Attribution License, which permits unrestricted use, distribution, and reproduction in any medium, provided the original work is properly cited.

\begin{abstract}
Alzheimer's (AD) and Parkinson's (PD) diseases are the two most common causes of dementia in aged population. Both are protein-misfolding diseases characterized by the presence of protein deposits in the brain. Despite growing evidence suggesting that oxidative stress is critical to neuronal death, its precise role in disease etiology and progression has not yet been fully understood. Budding yeast Saccharomyces cerevisiae shares conserved biological processes with all eukaryotic cells, including neurons. This fact together with the possibility of simple and quick genetic manipulation highlights this organism as a valuable tool to unravel complex and fundamental mechanisms underlying neurodegeneration. In this paper, we summarize the latest knowledge on the role of oxidative stress in neurodegenerative disorders, with emphasis on $\mathrm{AD}$ and $\mathrm{PD}$. Additionally, we provide an overview of the work undertaken to study $\mathrm{AD}$ and $\mathrm{PD}$ in yeast, focusing the use of this model to understand the effect of oxidative stress in both diseases.
\end{abstract}

\section{Introduction}

Misfolded proteins are typically insoluble and tend to form long linear or fibrillar aggregates known as amyloid deposits. Amyloid-like protein fibrils are a well-known pathological hallmark of age-related neurodegenerative diseases, including Alzheimer's disease (AD) and Parkinson's disease (PD). Alzheimer's and Parkinson's diseases are the most common forms of dementia, currently affecting 30 and 4 million people worldwide, respectively. In $\mathrm{AD}$, the beta-amyloid $(\mathrm{A} \beta)$ peptide accumulates mainly extracellularly, whereas in $\mathrm{PD}$, the $\alpha$-Synuclein $(\alpha$-Syn) protein accumulates, within neurons, inside the Lewy bodies (LB) and Lewy neurites (LN). Although there is a plethora of factors interfering in those pathological depositions, it is clear that oxidative stress may play a crucial role in neuronal death in neurodegenerative disorders [1-3].

Reactive oxygen species (ROS) are chemically reactive molecules containing oxygen and are produced in all aerobic cells. Oxidative stress occurs when the generation of ROS in a system exceeds that system's ability to neutralize and to eliminate them. All organisms have developed adaptive responses to oxidative stress that involve defensive enzyme and molecular chaperones - the expression of both being orchestrated by stress-responsive transcription factors-as well as antioxidant molecules [4]. Excessive production of ROS, and the consequent disruption of cellular redox balance, drives the oxidation of biological macromolecules, such as DNA, proteins, carbohydrates, and lipids, potentially leading to failure of biological functions [4].

Many ROS possess unpaired electrons and are therefore free radicals. The generation of free radicals is closely linked with the involvement of trace metals, particularly copper and irons $[5,6]$. To cope with this potential hazard, the concentration of cytosolic free metals is accurately controlled through regulation of their uptake, storage, and mobilization, in order to maintain redox-active metals in normal physiological limits $[7,8]$. Inside cells, "free pools" of copper and iron are avoided through their effective sequester by metal-binding proteins $[5,9]$. The chelatable redox-active 
iron constitutes the so-called labile iron pool (LIP), which serves as a transient source of iron $[5,9,10]$. Nevertheless, whenever cells are subjected to stress conditions, an excess of superoxide anion radical acts as an oxidant of Fe-S clusters of several enzymes, releasing "free iron." The released iron can in turn participate in Fenton type reactions, producing the highly reactive hydroxyl radical [11].

During the oxidative burst triggered during inflammatory processes, cells of the immune system produce both superoxide anion and nitric oxide (NO) free radicals. Nitric oxide is produced by the NO synthase family of enzymes. NO may directly react with its biological targets, as it is known to regulate the catalytic activity of various enzymes primarily by interacting with Fe-S clusters, oxidized copper centres, heme, and tyrosyl radicals [12]. NO also reacts with superoxide ion $\left(\mathrm{O}_{2}{ }^{-}\right)$or oxygen to form the nitrogen radical peroxynitrite $\left(\mathrm{ONOO}^{-}\right)$. Reactive nitrogen species (RNS) are highly reactive towards biological macromolecules and are thought to be responsible for NO-mediated cell death.

The aim of this paper is to provide an overview on the role of oxidative stress in neurodegenerative disorders, with emphasis on $\mathrm{AD}$ and $\mathrm{PD}$. Despite the absence of a nervous system in yeast, several studies have shown that this eukaryotic unicellular organism is a suitable model system to understand the molecular mechanisms underlying neurodegenerative diseases. The knowledge from those studies is summarized herein. Finally, we discuss how yeast models have been or may be used to extend our understanding on the role of oxidative stress in $\mathrm{AD}$ and PD.

\section{Oxidative Stress and Neurodegenerative Diseases}

The human brain is responsible for approximately $20 \%$ of our body oxygen consumption and thus subjected to a high metabolically derived level of ROS [13, 14]. An increasing body of evidence suggests that oxidative stress is involved in the etiology and pathogenesis of neurological disorders. The lipid bilayer of the brain is rich in polyunsaturated fatty acids and oxygen and is therefore highly susceptible to lipid peroxidation, a complex process involving the interaction of polyunsaturated fatty acids with free radicals that results in production of reactive electrophilic aldehydes. Lipid peroxidation occurs in several neurodegenerative diseases [15]. Evidence of oxidative stress in these diseases is further supported by increased DNA (and often RNA) base oxidation products and oxidative protein damages in specific regions of the brain [4]. The destruction of cellular components can induce a diversity of cellular responses through generation of secondary reactive species and ultimately lead to cell death via apoptosis and necrosis [1618]. Mitochondrion is the center of ROS production. About $90 \%$ of mammalian oxygen consumption is mitochondrial, making mitochondria particularly important in neurons due to their high demands for energy. This fact, together with the observation that mitochondrial perturbation occurs in multiple neurodegenerative disorders [19], suggests that neurodegenerative diseases are mitochondrial diseases.

\section{Alzheimer's Disease and Oxidative Stress}

Alzheimer's disease $(\mathrm{AD})$ is an age-related progressive neurodegenerative disease caused by severe neurodegeneration in the hippocampus and neocortical regions of the brain of affected individuals [20].

AD pathological hallmarks include extracellular amyloid plaques and intracellular aggregates (neurofibrillary tangles). The major component of the amyloid plaques is the amyloid peptide $\mathrm{A} \beta$, which results from the proteolysis of the amyloid precursor protein (APP). APP is a ubiquitously expressed transmembrane protein exerting a critical role in neuron growth and survival $[21,22]$. APP proteolysis to form the $\mathrm{A} \beta$ peptide involves the sequential action of aspartic protease BACE1 $(\beta$-secretase) and of $\gamma$-secretase, a multiprotein complex [23]. The length of $\mathrm{A} \beta$ peptide may range from 39 to 43 aminoacid residues, due to different $\gamma$-secretase cleavage sites. $\mathrm{A} \beta$ appears to be unfolded, under physiological conditions [24]. In amyloid plaques, the most frequent species are $A \beta 40$ and $A \beta 42$, the latter being the most prone to aggregation [23]. Neurofibrillary tangles are composed of hyperphosphorylated tau protein, a microtubule-binding protein thought to be involved in microtubules stabilization and in regulation of axonal transport in the brain [25].

The causes of Alzheimer's disease are not well understood, except for a small percentage of cases that are linked to familial genetic mutations [26]. Several hypotheses have been put forward with the aim to explain the cause of the sporadic form of the disease. One widely discussed of those hypotheses assumes that amyloid deposits of $\mathrm{A} \beta$ peptides are the causative agents of $\mathrm{AD}$ [27]. The "amyloid" theory is further supported by the link between mutations in the APP gene and some inherited forms of the disease [26].

$\mathrm{A} \beta$ toxicity is dependent on $\mathrm{A} \beta$ 's conformational state, peptide length, and concentration $[28,29]$. Moreover, it has been described that $\mathrm{A} \beta$ toxicity is also related to $\mathrm{A} \beta$ 's ability to form hydrogen peroxide and free radicals $[30,31]$. These findings are supported by the significant lipid peroxidation, protein oxidation, and DNA oxidation observed in AD brains $[29,32,33]$. In addition, two factors reinforce the role of oxidative stress in $\mathrm{AD}$ pathogenesis: pro-oxidants increase $\mathrm{A} \beta$ production, whereas several antioxidants, namely vitamin $\mathrm{E}$, melatonin, and several free radical scavengers, can protect neurons from $\mathrm{A} \beta$-induced toxicity [34].

Interestingly, the $\mathrm{A} \beta$ peptide is not toxic in the absence of redox metal ions, and many recent studies implicate biometals in the development or progression of Alzheimer's disease [6, 35-37]. Accordingly, sophisticated techniques have shown an overaccumulation of copper, iron, and zinc within the amyloid plaques compared with the surrounding tissues [38]. A $\beta$ has high affinity for redox-active metals being able to reduce them and consequently lead to the formation of hydrogen peroxide and oxidized amyloid [6]. Butterfield and Bush proposed that a single methionine residue (Met35) of $\mathrm{A} \beta 42$ is critical for the oxidative and 
neurotoxic properties of this peptide $[39,40]$. Substitution of Met 35 renders the $\mathrm{A} \beta$ peptide nonoxidative and nonneurotoxic [40]. The sulphur atom of Met35 is highly susceptible to oxidation, forming the sulfide radical MetS ${ }^{\bullet+}$ and reducing copper(II) to its high-active low-valency form [5, 40]. The $\mathrm{MetS}^{\bullet+}$ radical is able to undergo very fast reactions with superoxide ion, leading to the formation of methionine sulfoxide (MetO). In AD senile plaques, a significant fraction of $\mathrm{A} \beta$ has Met35 in the form of MetO [41].

Another well-studied source of oxidative stress in AD is mitochondria damage and its consequent functional abnormality that favors the production of ROS. Indeed, it was shown that neurons in $\mathrm{AD}$ exhibit a significantly higher percentage of damaged mitochondria compared to an aged-matched group [42]. Furthermore, mitochondrial dysfunction has been widely implicated in the etiology of $\mathrm{AD}$, since early impairments of mitochondrial function and oxidative stress may precede $\mathrm{A} \beta$ overproduction and deposition [43]. Also inflammation can induce oxidative damage in $\mathrm{AD}$, especially via microglia, leading to increased ROS and RNS formation and the resulting damage to lipid, proteins, and nucleic acids [44-46].

\section{Parkinson's Disease and Oxidative Stress}

Parkinson's disease is an age-related neurodegenerative disorder affecting the central nervous system. It is characterized by the progressive degeneration of nigrostriatal dopaminergic neurons within the substantia nigra pars compacta, which is the pathological process responsible for the motor symptoms attributed to PD [47]. The pathological hallmark of the disease is the presence of proteinaceous cytoplasmic inclusions designated as Lewy bodies and Lewy neurites. These are predominantly composed of the presynaptic protein $\alpha$-Synuclein ( $\alpha$-Syn) [48] together with proteasomal and lysosomal subunits as well as molecular chaperones [49].

The ubiquitous $\alpha$-Syn brain protein is implicated in both hereditary and sporadic PD. Its encoding gene, SNCA, was the first genetic determinant associated with the disease and, for this reason, much of the work on PD converges on $\alpha$-Syn [50]. $\alpha$-Syn was shown to interact with lipids and membranes, accelerating amyloid fibril formation [51], and it has been proposed to regulate the dynamics of synaptic vesicles at the synapse [52]. Indeed, $\alpha$-Syn exhibits a remarkable conformational plasticity being its structure largely dependent on the surrounding environment. The monomeric $\alpha$-Syn is a typical natively unfolded protein under physiological conditions [53, 54]. However, under specific conditions, such as the increase of its intracellular levels, $\alpha$-Syn can adopt different conformations, including several $\alpha$-helical and $\beta$-sheet species folded to different degrees in both monomeric and oligomeric states [55].

Although PD is a recognized multifactorial disease, a large body of evidence has implicated oxidative stress in the pathogenesis of PD. The conclusive connection between PD and oxidative stress is supported by the increased oxidative damage of sugars, lipids, nucleic acids, and proteins observed in postmortem dopaminergic neurons within the substantia nigra pars compacta of PD brains $[6,56,57]$.
Auluck et al. proposed that the impairment of $\alpha$ Syn function leads to its local accumulation, favoring the formation of toxic oligomeric species that interfere with ER-to-Golgi trafficking, mitochondria turnover-through the abrogation of mitophagy — and generate oxidative stress. Moreover, the abnormal interaction of $\alpha$-Syn with membranes has been implicated in the cytoplasmic retention of catecholaminergic neurotransmitters yielding cytotoxicity through the generation of dopamine adducts and ROS [52]. This effect is potentiated in the presence of iron-rich environments, as it is the case of Lewis bodies in the neurons decorating the substantia nigra of PD patients [58-61]. Indeed, it is known that dopamine is able to coordinate iron and regenerate $\mathrm{Fe}^{2+}$, possibly providing an equally important source of hydroxyl radical production [62].

Mitochondria have been claimed as dominant sites for oxidative stress-driven initiation and propagation in PD. The direct implication of this organelle in PD was first suggested by the use of the mitochondrial complex I (CI) inhibitor MPTP (1-methyl-4-phenyl-1,2,3,6-tetrahydropyridine) [63, 64]. This chemical mimics human PD in animal models and is associated with development of Parkinsonism in humans subjected to accidental exposure [65]. Further corroborating the relevance of mitochondria in $\mathrm{PD}$, it was shown that the well-known CI inhibitor rotenone induces death of dopaminergic cells [66-69]. In addition, depletion of the antioxidant peptide glutathione (GSH) in PD cells, which may be caused by a decrease in its synthesis and recycling [70], has been associated with a decrease of mitochondrial CI activity, resulting in mitochondrial dysfunction [71, 72]. Moreover, defective mitochondrial CI function is observed in the substantia nigra of PD brains $[73,74]$. Recent studies have also demonstrated that $\alpha$-Syn monomers and oligomers associate with the inner mitochondrial membrane where they can physically associate with CI, thereby interfering with the mitochondrial function and increasing free radicals production [72, 75]. Further highlighting the relevance of mitochondrial dysfunction and oxidative stress in the pathological process of the disease, several genes associated with familial cases of PD were shown to encode either mitochondrial proteins or mitochondria-associated proteins $[6$, 76]. Among the latter is DJ-1, a protein that shares structural homology with the Escherichia coli chaperone Hsp31 and is thought to have a protective role against oxidative stress [77]. Under conditions of oxidative stress, DJ-1 is relocated to the mitochondria, affecting the sensitivity of specific neuron populations to compounds targeting mitochondrial CI, namely, rotenone, paraquat, and MPTP [6, 78-80].

Several evidences support as well an unbalanced generation of RNS as a feature of PD pathology. First, nitrated $\alpha$ Syn accumulates in LB of PD cells. Secondly, the treatment of GSH-depleted dopaminergic cells with RNS inhibitors prevents mitochondrial CI inhibition [6], indicating that RNS itself has a role in mitochondrial dysfunction and ROS generation in PD. Lastly, glial cells within the substantia nigra exhibit increased NO levels [81], possibly due to the accumulation of interferon- $\gamma$ (IFN- $\gamma$ ) [82], a cytokine which was shown to promote induction of RNS in brain. 


\section{S. cerevisiae as Model Organism to Study Neurodegenerative Diseases}

Budding yeast Saccharomyces cerevisiae has been introduced as an experimental organism in the midthirties of the 20 century. Since then, its potential as a model organism has been exploited in many areas of biology [83]. Despite lacking the physiological complexity of the mammalian nervous system, yeast was recently used in the study of neurodegenerative disorders, such as Alzheimer's and Parkinson's Diseases. This became possible due to the development of powerful yeast genetic tools as well as the high conservation of fundamental biological processes and pathways associated with neurodegeneration including protein folding, cellular trafficking, and secretion [84]. It is noteworthy that about one-fifth of yeast genes are members of orthologous gene families associated with human diseases [85]. This is an important aspect to consider when studying human diseases in yeast. If a homologue of the gene implicated in the disease is present in yeast genome, a unique opportunity to directly study its function is offered, either through its deletion or overexpression. Otherwise, if the disease-associated gene does not have a yeast counterpart, functional analysis can still be performed via heterologous expression [86, 87]. Equally important "humanized" yeasts are being used as platforms for high-throughput screenings of compounds with therapeutic potential.

\section{Yeast as a Model for Studying Alzheimer's Disease}

Yeast models have been extensively used to study several molecular aspects of $\mathrm{AD}$, even though yeast lacks for some AD-associated genes. Studies in yeast have been mainly focused on the in vivo APP processing, A $\beta$ oligomerization, and toxicity.

The usage of heterologous expression of human secretases in yeast has greatly contributed to the understanding of human APP processing. It has allowed the discovery of BACE1 inhibitors and prompted the study of the individual function of each component of the $\gamma$-secretase complex [88].

Growing evidence suggests that the oligomeric forms of the $\mathrm{A} \beta$ peptide, rather than amyloid fibrils, are the most toxic forms [89-92]. These findings have shifted the focus of investigation towards the earliest stages of $\mathrm{A} \beta$ oligomerization. As a result, the following described yeast systems were developed and are now useful tools not only in the study of $\mathrm{A} \beta$-oligomerization, but also in the understanding of the molecular events triggered by aggregation as well as in the screening of potential therapeutic compounds that affect the aggregation process.

The first yeast study on $\mathrm{A} \beta$ oligomerization used a twohybrid approach to analyze $\mathrm{A} \beta$ dimerization. Protein-protein interactions were measured by fusing the $\mathrm{A} \beta$ peptide to a LexA DNA-binding domain and also to a B42 transactivation domain, and then monitoring the expression of a lac $Z$ reporter driven by a LexA-dependent promoter [93]. The authors showed that $\mathrm{A} \beta$ peptide was able to form dimers, in vivo, in the yeast cell nucleus.
Bagriantsev and Liebman and von der Haar et al. implemented a different yeast model system that may constitute a valuable tool to seek for agents that interfere with the initial steps of $\mathrm{A} \beta 42$ oligomerization $[94,95]$. In this study, the ability of $\mathrm{A} \beta 42$ peptide to aggregate was monitored by fusing it to the middle and C-terminal domain of Sup35. The Sup35 yeast translational termination factor can undergo spontaneous conversion into a prion state, losing its function [96]. Sup35 loses the ability to aggregate when its prion-forming (N-terminal) domain is deleted. However, the insertion of $\mathrm{A} \beta$ peptide sequences in place of the original prion domain of Sup35 protein restores its ability to aggregate $[94,95]$. Using this reporter system, it was possible to confirm in vivo the impact of point mutations, previously shown to inhibit A $\beta 42$ aggregation in vitro [94, 97]. Furthermore, it was shown that the Hsp104 yeast chaperone, a chaperone known to rescue proteins from the aggregated state in other yeast models of neurodegenerative diseases [98, 99], appears to have a contrary function in $\mathrm{AD}$, protecting $\mathrm{A} \beta$-fusion protein from disaggregation and degradation $[94,95]$.

Oligomerization of $\mathrm{A} \beta$ was also the subject of a third yeast study, by the use of a reporter consisting of $A \beta$ fragment fused to GFP [100]. The assay was based on the premise that aggregates of the fusion protein suppressed green fluorescence. The A $\beta$-GFP fusion was shown to cause slight but significant yeast growth reduction and to induce a heat shock response (HSR), as indicated by the cotransformation of yeast with $\mathrm{A} \beta$-GFP and HSE2 element fused to a downstream lac $Z$ gene. The authors put forward the hypothesis that HSR could arise from $\mathrm{A} \beta$ inducing ROS and/or the presence of misfolded proteins and suggested that HSR might be a target for further studies seeking for inhibitors of $A \beta$ effects [100].

Recently, Treusch et al. engineered a yeast model for studying $\mathrm{A} \beta$ toxicity [101]. The overexpression of a construct harboring the $\mathrm{A} \beta 42$ fragment fused at the $\mathrm{N}$-terminus to an endoplasmatic reticulum targeting sequence was driven by a galactose-inducible promoter. $\mathrm{A} \beta$ oligomers localized to secretory compartments and, like in neurons, contributed to toxicity in yeast. A screen for genetic modifiers allowed the identification of 40 genes that were able to modulate $\mathrm{A} \beta$ toxicity. Among those, 12 had homologues in humans, 3 being related to clathrin-mediated endocytosis, and 7 functionally associated with the cytoskeleton. Interestingly, all the former genes behaved as $\mathrm{A} \beta$ toxicity suppressors and had been previously shown to be or interact with validated risk factors for $\mathrm{AD}$. The authors further showed that $\mathrm{A} \beta$ affects clathrin-mediated endocytosis and proposed that $\mathrm{A} \beta$ oligomers may interact with transmembranar receptors and prevent their correct destination [101].

\section{Yeast as a Model for Studying Parkinson's Disease}

As a common feature of sporadic and familial cases of PD, the understanding of the pathological processes associated with $\alpha$-Syn has attracted special attention. In order to gain insight into $\alpha$-Syn pathobiology, Outeiro and Lindquist 
exploited a myriad of advantages of using S. cerevisiae as a model organism, by developing a powerful "humanized" yeast system. As a means to study the $\alpha$-Syn dynamics in vivo, the authors overexpressed in yeast cells a construct harboring the wild type or the mutant versions of human SNCA gene fused to GFP [102]. This pioneering system faithfully reproduces several features of PD in yeast, allowing to thoroughly investigate the pathological processes involved in the disease. Three strains, designated as NonTox, InTox, and HiTox, were created to express $\alpha$-Syn at different levels [52]. As it happens in complex eukaryotic models, the appearance of cytoplasmic foci, cytotoxicity, and $\alpha$ Syn-decorated vesicle accumulation was shown to be dose dependent $[102,103]$. Moreover, high doses of $\alpha$-Syn lead to increased toxicity, accumulation of cytoplasmic lipid droplets, vesicle trafficking defects, ER stress, activation of the heat-shock response, impairment of the ubiquitinproteasome pathway, and mitochondrial dysfunction in the HiTox strain, therefore recapitulating the pathological features displayed by PD patients whose genome encodes duplications or triplications of SNCA locus [52].

The $\alpha$-Syn yeast model developed by Outeiro and Lindquist has been the basis of several genome wide and high-throughput analyses aimed at unveiling the intricacies of PD. The systematic screening of a galactose-inducible overexpression library in the InTox strain revealed the Rab GTPase Ypt1 (Rab1) as suppressor of $\alpha$-Syn toxicity [104], reinforcing the role of $\alpha$-Syn in vesicle formation and delivery. In addition, an unbiased genome-wide screen for modifiers of $\alpha$-Syn toxicity was performed in the InTox strain, allowing the identification of the polyamine transporter Tpo4 [105] and highlighting the significance of polyamine pathway in PD pathogenesis. Using the ResponseNet algorithm to integrate $\alpha$-Syn mRNA profiling and genome-wide genetic data, it was found that trehalose might be involved in the protection pathway against $\alpha$-Syn toxicity possibly promoting misfolded protein clearance. In addition, mitochondrial dysfunction and oxidative/nitrosative stress also appeared as consequences of $\alpha$-Syn overexpression in yeast [106]. Comparison of the transcriptome of HiTox and NonTox strains provides further evidence supporting the assumption that mitochondrial dysfunction and oxidative stress are associated to conditions in which $\alpha$-Syn is expressed at high levels. It has also been verified that mitochondria morphology is affected and ROS is accumulated in the HiTox strain further suggesting that high levels of $\alpha$-Syn elicit global mitochondrial dysfunction [107]. This may suggest that $\alpha$-Syn accumulation is the origin of oxidative damage of specific neuronal cells in PD.

More recently, overexpression of $\alpha$-Syn in yeast revealed that the knockout of genes encoding lipid elongases, namely, ELO1, ELO2, and ELO3, impairs cell growth, dramatically decreases the survival of aged cells, and leads also to ROS accumulation and aberrant protein trafficking [108]. A similar strategy, using a different plasmid to drive galactoseinducible $\alpha$-Syn expression in distinct $S$. cerevisiae backgrounds, disclosed the significance of fatty acid synthase activity and intracellular redox status in the mechanisms of $\alpha$-Syn toxicity [109].
$\alpha$-Syn-humanized yeasts have also been exploited to search for compounds with therapeutic potential. In this context, the HiTox strain was used in a high-throughput chemical screen to identify agents capable of rescuing the robust toxicity of this strain. A class of small molecules of 1,2,3,4-tetrahydroquinolinones were identified and shown to revert the formation of $\alpha$-Syn foci, to reestablish ER-to-Golgi trafficking, to ameliorate mitochondria damage, to limit ROS production, and consequently to reduce $\alpha$-Syn toxicity not only in yeast but also in other more complex PD models [107].

\section{Concluding Remarks}

Although $\mathrm{AD}$ and $\mathrm{PD}$ have been extensively studied, the exact mechanism of disease progression or pathogenesis remains largely unknown. As outlined in this paper, several in vivo and in vitro studies point towards a role of oxidative stress in $\mathrm{AD}$ and $\mathrm{PD}$ pathogenesis. Nevertheless, whether it is a primary cause or simply a consequence of the neurodegenerative process is still an unanswered question. In addition, specifically concerning $\mathrm{AD}$, there are quite a few contradictory reports regarding the role of oxidative stress in the disease. Indeed, it has been described that oxidative stress may as well lead to an increase in $\mathrm{A} \beta[14,110]$, and in vivo studies showed a negative correlation between oxidative stress and $\mathrm{A} \beta$, indicating an antioxidant role for $\mathrm{A} \beta$ [111].

Yeast can be a powerful tool as a means to clarify several of these issues. Within this context, yeast models of AD may in the future be used to monitor $\mathrm{A} \beta$ oligomerization and toxicity under an oxidative environment or in the absence of ROS (hypoxia). Interestingly, a yeast model consisting of $\mathrm{A} \beta$ peptide fused to GFP has been successfully used to test whether folate, an antioxidant, was able to prevent $\mathrm{A} \beta$ aggregation [112]. To better understand the relationship between oxidative stress and $\alpha$-Syn aggregation, in the pathological processes triggering $\mathrm{PD}$, it would be interesting to assess both the behavior of $\alpha$-Syn in the "humanized" NonTox strain under oxidative environments and in the InTox and HiTox strains under hypoxia conditions.

Future studies combining yeast and animal models of AD and PD will certainly provide valuable insights into the role of oxidative stress in these neurodegenerative diseases.

\section{Acknowledgments}

This work was supported by Fundação para a Ciência e a Tecnologia (FCT) through Grants PTDC/BIA-MIC/108747/ 2008 and Pest-OE/EQB/LA0004/2011. C. Pimentel (SFRH/ BPD/35052/2007), L. Batista-Nascimento (SFRH/BD/39389/ 2007), and R. A. Menezes (SFRH/BPD/26506/2006) were supported by FCT fellowships.

\section{References}

[1] P. Mao and P. H. Reddy, "Is multiple sclerosis a mitochondrial disease?" Biochimica et Biophysica Acta, vol. 1802, no. 1, pp. 66-79, 2010. 
[2] A. A. Starkov, C. Chinopoulos, and G. Fiskum, "Mitochondrial calcium and oxidative stress as mediators of ischemic brain injury," Cell Calcium, vol. 36, no. 3-4, pp. 257-264, 2004.

[3] M. Valko, D. Leibfritz, J. Moncol, M. T. D. Cronin, M. Mazur, and J. Telser, "Free radicals and antioxidants in normal physiological functions and human disease," International Journal of Biochemistry and Cell Biology, vol. 39, no. 1, pp. 44-84, 2007.

[4] M. Ramalingam and S. J. Kim, "Reactive oxygen/nitrogen species and their functional correlations in neurodegenerative diseases," Journal of Neural Transmission. In press.

[5] K. Jomova and M. Valko, "Advances in metal-induced oxidative stress and human disease," Toxicology, vol. 283, no. 2-3, pp. 65-87, 2011.

[6] K. Jomova, D. Vondrakova, M. Lawson, and M. Valko, "Metals, oxidative stress and neurodegenerative disorders," Molecular and Cellular Biochemistry, vol. 345, no. 1-2, pp. 91$104,2010$.

[7] M. W. Hentze, M. U. Muckenthaler, B. Galy, and C. Camaschella, "Two to tango: regulation of mammalian iron metabolism,” Cell, vol. 142, no. 1, pp. 24-38, 2010.

[8] B. E. Kim, T. Nevitt, and D. J. Thiele, "Mechanisms for copper acquisition, distribution and regulation," Nature Chemical Biology, vol. 4, no. 3, pp. 176-185, 2008.

[9] K. Jomova and M. Valko, "Importance of iron chelation in free radical-induced oxidative stress and human disease," Current Pharmaceutical Design, vol. 17, no. 31, pp. 34603473, 2011.

[10] O. Kakhlon and Z. I. Cabantchik, "The labile iron pool: characterization, measurement, and participation in cellular processes," Free Radical Biology and Medicine, vol. 33, no. 8, pp. 1037-1046, 2002.

[11] R. Meneghini, "Iron homeostasis, oxidative stress, and DNA damage," Free Radical Biology and Medicine, vol. 23, no. 5, pp. 783-792, 1997.

[12] M. C. Carreras, M. C. Franco, J. G. Peralta, and J. J. Poderoso, "Nitric oxide, complex I, and the modulation of mitochondrial reactive species in biology and disease," Molecular Aspects of Medicine, vol. 25, no. 1-2, pp. 125-139, 2004.

[13] R. G. Shulman, D. L. Rothman, K. L. Behar, and F. Hyder, "Energetic basis of brain activity: implications for neuroimaging," Trends in Neurosciences, vol. 27, no. 8, pp. 489-495, 2004.

[14] B. Su, X. Wang, A. Nunomura et al., "Oxidative stress signaling in Alzheimer's disease," Current Alzheimer Research, vol. 5, no. 6, pp. 525-532, 2008.

[15] T. T. Reed, "Lipid peroxidation and neurodegenerative disease," Free Radical Biology \& Medicine, vol. 51, no. 7, pp. 1302-1319, 2011.

[16] J. A. Klein and S. L. Ackerman, "Oxidative stress, cell cycle, and neurodegeneration," Journal of Clinical Investigation, vol. 111, no. 6, pp. 785-793, 2003.

[17] B. Palmieri and V. Sblendorio, "Oxidative stress tests: overview on reliability and use-Part I," European Review for Medical and Pharmacological Sciences, vol. 11, no. 5, pp. 309342, 2007.

[18] K. Kannan and S. K. Jain, "Oxidative stress and apoptosis," Pathophysiology, vol. 7, no. 3, pp. 153-163, 2000.

[19] H. Du and S. S. Yan, "Mitochondrial medicine for neurodegenerative diseases," The International Journal of Biochemistry \& Cell Biology, vol. 42, no. 5, pp. 560-572, 2010.
[20] H. Braak, E. Braak, D. Yilmazer, R. A. I. de Vos, E. N. H. Jansen, and J. Bohl, "Pattern of brain destruction in Parkinson's and Alzheimer's diseases," Journal of Neural Transmission, vol. 103, no. 4, pp. 455-490, 1996.

[21] C. Priller, T. Bauer, G. Mitteregger, B. Krebs, H. A. Kretzschmar, and J. Herms, "Synapse formation and function is modulated by the amyloid precursor protein," The Journal of Neuroscience, vol. 26, no. 27, pp. 7212-7221, 2006.

[22] P. R. Turner, K. O'Connor, W. P. Tate, and W. C. Abraham, "Roles of amyloid precursor protein and its fragments in regulating neural activity, plasticity and memory," Progress in Neurobiology, vol. 70, no. 1, pp. 1-32, 2003.

[23] R. J. O’Brien and P. C. Wong, "Amyloid precursor protein processing and Alzheimer's disease," Annual Review of Neuroscience, vol. 34, pp. 185-204, 2011.

[24] V. N. Uversky, "Intrinsic disorder in proteins associated with neurodegenerative diseases," Frontiers in Bioscience, vol. 14, no. 14, pp. 5188-5238, 2009.

[25] V. M. Y. Lee, M. Goedert, and J. Q. Trojanowski, "Neurodegenerative tauopathies," Annual Review of Neuroscience, vol. 24, pp. 1121-1159, 2001.

[26] D. Avramopoulos, "Genetics of Alzheimer's disease: recent advances," Genome Medicine, vol. 1, no. 3, p. 34, 2009.

[27] J. Hardy and D. Allsop, "Amyloid deposition as the central event in the aetiology of Alzheimer's disease," Trends in Pharmacological Sciences, vol. 12, no. 10, pp. 383-388, 1991.

[28] L. L. Iversen, R. J. Mortishire-Smith, S. J. Pollack, and M. S. Shearman, "The toxicity in vitro of $\beta$-amyloid protein," Biochemical Journal, vol. 311, pp. 1-16, 1995.

[29] A. Kontush, "Amyloid- $\beta$ : an antioxidant that becomes a prooxidant and critically contributes to Alzheimer's disease," Free Radical Biology and Medicine, vol. 31, no. 9, pp. 11201131, 2001.

[30] B. J. Tabner, S. Turnbull, O. El-Agnaf, and D. Allsop, "Production of reactive oxygen species from aggregating proteins implicated in Alzheimer's disease, Parkinson's disease and other neurodegenerative diseases," Current Topics in Medicinal Chemistry, vol. 1, no. 6, pp. 507-517, 2001.

[31] B. J. Tabner, S. Turnbull, O. M. A. El-Agnaf, and D. Allsop, "Formation of hydrogen peroxide and hydroxyl radicals from A $\beta$ and $\alpha$-synuclein as a possible mechanism of cell death in Alzheimer's disease and Parkinson's disease," Free Radical Biology and Medicine, vol. 32, no. 11, pp. 1076-1083, 2002.

[32] A. Nunomura, T. Hofer, P. I. Moreira, R. J. Castellani, M. A. Smith, and G. Perry, "RNA oxidation in Alzheimer disease and related neurodegenerative disorders," Acta Neuropathologica, vol. 118, no. 1, pp. 151-166, 2009.

[33] D. R. D. Premkumar, M. A. Smith, P. L. Richey et al., "Induction of heme oxygenase-1 mRNA and protein in neocortex and cerebral vessels in Alzheimer's disease," Journal of Neurochemistry, vol. 65, no. 3, pp. 1399-1402, 1995.

[34] M. Dumont and M. F. Beal, "Neuroprotective strategies involving ROS in Alzheimer disease," Free Radical Biology and Medicine, vol. 51, no. 5, pp. 1014-1026, 2011.

[35] F. Molina-Holgado, R. C. Hider, A. Gaeta, R. Williams, and P. Francis, "Metals ions and neurodegeneration," BioMetals, vol. 20, no. 3-4, pp. 639-654, 2007.

[36] G. A. Salvador, R. M. Uranga, and N. M. Giusto, "Iron and mechanisms of neurotoxicity," International Journal of Alzheimer's Disease, vol. 2010, Article ID 720658, 9 pages, 2011.

[37] Y. H. Hung, A. I. Bush, and R. A. Cherny, "Copper in the brain and Alzheimer's disease," Journal of Biological Inorganic Chemistry, vol. 15, no. 1, pp. 61-76, 2010. 
[38] R. Rajendran, R. Minqin, M. D. Ynsa et al., "A novel approach to the identification and quantitative elemental analysis of amyloid deposits-Insights into the pathology of Alzheimer's disease," Biochemical and Biophysical Research Communications, vol. 382, no. 1, pp. 91-95, 2009.

[39] D. A. Butterfield and A. I. Bush, "Alzheimer's amyloid $\beta$ peptide (1-42): Involvement of methionine residue 35 in the oxidative stress and neurotoxicity properties of this peptide," Neurobiology of Aging, vol. 25, no. 5, pp. 563-568, 2004.

[40] D. A. Butterfield and R. Sultana, "Methionine-35 of A $\beta(1-$ 42): importance for oxidative stress in Alzheimer disease," Journal of Amino Acids, vol. 2011, Article ID 198430, 10 pages, 2011.

[41] J. Naslund, A. Schierhorn, U. Hellman et al., "Relative abundance of Alzheimer $\mathrm{A} \beta$ amyloid peptide variants in Alzheimer disease and normal aging," Proceedings of the National Academy of Sciences of the United States of America, vol. 91, no. 18, pp. 8378-8382, 1994.

[42] K. Hirai, G. Aliev, A. Nunomura et al., "Mitochondrial abnormalities in Alzheimer's disease," Journal of Neuroscience, vol. 21, no. 9, pp. 3017-3023, 2001.

[43] D. F. F. Silva, A. R. Esteves, C. R. Oliveira, and S. M. Cardoso, "Mitochondria: the common upstream driver of amyloid- $\beta$ and tau pathology in Alzheimer's disease," Current Alzheimer Research, vol. 8, no. 5, pp. 563-572, 2011.

[44] B. Cameron and G. E. Landreth, "Inflammation, microglia, and Alzheimer's disease," Neurobiology of Disease, vol. 37, no. 3, pp. 503-509, 2010.

[45] P. Eikelenboom, E. van Exel, J. J. M. Hoozemans, R. Veerhuis, A. J. M. Rozemuller, and W. A. Van Gool, "Neuroinflammation - an early event in both the history and pathogenesis of Alzheimer's disease," Neurodegenerative Diseases, vol. 7, no. 1-3, pp. 38-41, 2010.

[46] P. Eikelenboom and R. Veerhuis, "The role of complement and activated microglia in the pathogenesis of Alzheimer's disease," Neurobiology of Aging, vol. 17, no. 5, pp. 673-680, 1996.

[47] P. Jenner, Hunot, Olanow et al., "Oxidative stress in Parkinson's disease," Annals of Neurology, vol. 53, supplement 3, pp. S26-S38, 2003.

[48] M. G. Spillantini, M. L. Schmidt, V. M. Y. Lee, J. Q. Trojanowski, R. Jakes, and M. Goedert, " $\alpha$-synuclein in Lewy bodies," Nature, vol. 388, no. 6645, pp. 839-840, 1997.

[49] M. C. Irizarry, W. Growdon, T. Gomez-Isla et al., "Nigral and cortical Lewy bodies and dystrophic nigral neurites in Parkinson's disease and cortical Lewy body disease contain $\alpha$ synuclein immunoreactivity," Journal of Neuropathology and Experimental Neurology, vol. 57, no. 4, pp. 334-337, 1998.

[50] M. H. Polymeropoulos, C. Lavedan, E. Leroy et al., "Mutation in the $\alpha$-synuclein gene identified in families with Parkinson's disease," Science, vol. 276, no. 5321, pp. 2045-2047, 1997.

[51] E. Jo, J. McLaurin, C. M. Yip, P. St. George-Hyslop, and P. E. Fraser, " $\alpha$-synuclein membrane interactions and lipid specificity," Journal of Biological Chemistry, vol. 275, no. 44, pp. 34328-34334, 2000.

[52] P. K. Auluck, G. Caraveo, and S. Lindquist, " $\alpha$-synuclein: membrane interactions and toxicity in parkinson's disease," Annual Review of Cell and Developmental Biology, vol. 26, pp. 211-233, 2010.

[53] B. C. McNulty, G. B. Young, and G. J. Pielak, "Macromolecular crowding in the Escherichia coli periplasm maintains $\alpha$ synuclein disorder," Journal of Molecular Biology, vol. 355, no. 5, pp. 893-897, 2006.
[54] V. N. Uversky, J. Li, and A. L. Fink, "Evidence for a partially folded intermediate in $\alpha$-synuclein fibril formation," Journal of Biological Chemistry, vol. 276, no. 14, pp. 10737-10744, 2001.

[55] V. N. Uversky, "Neuropathology, biochemistry, and biophysics of $\alpha$-synuclein aggregation," Journal of Neurochemistry, vol. 103, no. 1, pp. 17-37, 2007.

[56] P. Jenner and C. W. Olanow, "Oxidative stress and the pathogenesis of Parkinson's disease," Neurology, vol. 47, supplement 6, pp. S161-S170, 1996.

[57] G. Perry, J. Avila, M. G. Espey et al., "Biochemistry of neurodegeneration,” Science, vol. 291, no. 5504, pp. 595-597, 2001.

[58] L. M. Sayre, P. I. Moreira, M. A. Smith, and G. Perry, "Metal ions and oxidative protein modification in neurological disease," Annali dell'Istituto Superiore di Sanita, vol. 41, no. 2, pp. 143-164, 2005.

[59] R. Castellani, M. A. Smith, P. L. Richey, and G. Perry, "Glycoxidation and oxidative stress in Parkinson disease and diffuse Lewy body disease," Brain Research, vol. 737, no. 1-2, pp. 195-200, 1996.

[60] K. Jellinger, E. Kienzl, G. Rumpelmair et al., "Iron-melanin complex in substantia nigra of Parkinsonian brains: an x-ray microanalysis," Journal of Neurochemistry, vol. 59, no. 3, pp. 1168-1171, 1992.

[61] R. J. Castellani, S. L. Siedlak, G. Perry, and M. A. Smith, "Sequestration of iron by Lewy bodies in Parkinson's disease," Acta Neuropathologica, vol. 100, no. 2, pp. 111-114, 2000.

[62] D. Ben-Shachar and M. B. H. Youdim, "Iron, melanin and dopamine interaction: relevance to Parkinson's disease," Progress in Neuro-Psychopharmacology and Biological Psychiatry, vol. 17, no. 1, pp. 139-150, 1993.

[63] M. T. Lin and M. F. Beal, "Mitochondrial dysfunction and oxidative stress in neurodegenerative diseases," Nature, vol. 443, no. 7113, pp. 787-795, 2006.

[64] C. Zhou, Y. Huang, and S. Przedborski, "Oxidative stress in Parkinson's disease: a mechanism of pathogenic and therapeutic significance," Annals of the New York Academy of Sciences, vol. 1147, pp. 93-104, 2008.

[65] J. W. Langston, P. Ballard, J. W. Tetrud, and I. Irwin, “Chronic parkinsonism in humans due to a product of meperidineanalog synthesis," Science, vol. 219, no. 4587, pp. 979-980, 1983.

[66] C. M. Testa, T. B. Sherer, and J. T. Greenamyre, "Rotenone induces oxidative stress and dopaminergic neuron damage in organotypic substantia nigra cultures," Molecular Brain Research, vol. 134, no. 1, pp. 109-118, 2005.

[67] T. B. Sherer, R. Betarbet, C. M. Testa et al., "Mechanism of toxicity in rotenone models of Parkinson's disease," Journal of Neuroscience, vol. 23, no. 34, pp. 10756-10764, 2003.

[68] G. U. Höglinger, W. H. Oertel, and E. C. Hirsch, "The rotenone model of Parkinsonism-the five years inspection," Journal of Neural Transmission, Supplement, no. 70, pp. 269272, 2006.

[69] T. B. Sherer, J. H. Kim, R. Betarbet, and J. T. Greenamyre, "Subcutaneous rotenone exposure causes highly selective dopaminergic degeneration and $\alpha$-synuclein aggregation," Experimental Neurology, vol. 179, no. 1, pp. 9-16, 2003.

[70] H. L. Martin and P. Teismann, "Glutathione-a review on its role and significance in Parkinson's disease," The FASEB Journal, vol. 23, no. 10, pp. 3263-3272, 2009.

[71] K. F. Winklhofer and C. Haass, "Mitochondrial dysfunction in Parkinson's disease," Biochimica et Biophysica Acta, vol. 1802, no. 1, pp. 29-44, 2010. 
[72] S. J. Chinta and J. K. Andersen, "Redox imbalance in Parkinson's disease," Biochimica et Biophysica Acta, vol. 1780, no. 11, pp. 1362-1367, 2008.

[73] A. H. Schapira, "Mitochondria in the aetiology and pathogenesis of Parkinson's disease," The Lancet Neurology, vol. 7, no. 1, pp. 97-109, 2008.

[74] A. H. V. Schapira, J. M. Cooper, D. Dexter, P. Jenner, J. B. Clark, and C. D. Marsden, "Mitochondrial complex I deficiency in Parkinson's disease," The Lancet, vol. 1, no. 8649, p. 1269, 1989.

[75] S. J. Chinta, J. K. Mallajosyula, A. Rane, and J. K. Andersen, "Mitochondrial $\alpha$-synuclein accumulation impairs complex I function in dopaminergic neurons and results in increased mitophagy in vivo," Neuroscience Letters, vol. 486, no. 3, pp. 235-239, 2010.

[76] G. E. Gibson, A. Starkov, J. P. Blass, R. R. Ratan, and M. F. Beal, "Cause and consequence: mitochondrial dysfunction initiates and propagates neuronal dysfunction, neuronal death and behavioral abnormalities in age-associated neurodegenerative diseases," Biochimica et Biophysica Acta, vol. 1802, no. 1, pp. 122-134, 2010.

[77] B. Alvarez-Castelao, C. Muñoz, I. Sánchez, M. Goethals, J. Vandekerckhove, and J. G. Castaño, "Reduced protein stability of human DJ-1/PARK7 L166P, linked to autosomal recessive Parkinson disease, is due to direct endoproteolytic cleavage by the proteasome," Biochimica et Biophysica Acta, vol. 1823, no. 2, pp. 524-533, 2012.

[78] R. M. Canet-Avilés, M. A. Wilson, D. W. Miller et al., “The Parkinson's disease DJ-1 is neuroprotective due to cysteinesulfinic acid-driven mitochondrial localization," Proceedings of the National Academy of Sciences of the United States of America, vol. 101, no. 24, pp. 9103-9108, 2004.

[79] H. J. Kwon, J. Y. Heo, J. H. Shim et al., "DJ-1 mediates paraquat-induced dopaminergic neuronal cell death," Toxicology Letters, vol. 202, no. 2, pp. 85-92, 2011.

[80] K. J. Thomas, M. K. McCoy, J. Blackinton et al., "DJ-1 acts in parallel to the PINK1/parkin pathway to control mitochondrial function and autophagy," Human Molecular Genetics, vol. 20, no. 1, pp. 40-50, 2011.

[81] S. Hunot, F. Boissière, B. Faucheux et al., "Nitric oxide synthase and neuronal vulnerability in Parkinson's disease," Neuroscience, vol. 72, no. 2, pp. 355-363, 1996.

[82] G. Boka, P. Anglade, D. Wallach, F. Javoy-Agid, Y. Agid, and E. C. Hirsch, "Immunocytochemical analysis of tumor necrosis factor and its receptors in Parkinson's disease," Neuroscience Letters, vol. 172, no. 1-2, pp. 151-154, 1994.

[83] D. Botstein and G. R. Fink, "Yeast: an experimental organism for 21st century biology," Genetics, vol. 189, no. 3, pp. 695704, 2011.

[84] L. Miller-Fleming, F. Giorgini, and T. F. Outeiro, "Yeast as a model for studying human neurodegenerative disorders," Biotechnology Journal, vol. 3, no. 3, pp. 325-338, 2008.

[85] S. Heinicke, M. S. Livstone, C. Lu et al., "The Princeton Protein Orthology Database (P-POD): a comparative genomics analysis tool for biologists," PLoS ONE, vol. 2, no. 8, p. e766, 2007.

[86] S. Tenreiro and T. F. Outeiro, "Simple is good: yeast models of neurodegeneration," FEMS Yeast Research, vol. 10, no. 8, pp. 970-979, 2010.

[87] V. Khurana and S. Lindquist, "Modelling neurodegeneration in Saccharomyces cerevisiae why cook with baker's yeast?" Nature Reviews Neuroscience, vol. 11, no. 6, pp. 436-449, 2010.
[88] P. Bharadwaj, R. Martins, and I. Macreadie, "Yeast as a model for studying Alzheimer's disease," FEMS Yeast Research, vol. 10, no. 8, pp. 961-969, 2010.

[89] D. M. Walsh, B. P. Tseng, R. E. Rydel, M. B. Podlisny, and D. J. Selkoe, "The oligomerization of amyloid $\beta$-protein begins intracellularly in cells derived from human brain," Biochemistry, vol. 39, no. 35, pp. 10831-10839, 2000.

[90] G. M. Shankar, S. Li, T. H. Mehta et al., "Amyloid- $\beta$ protein dimers isolated directly from Alzheimer's brains impair synaptic plasticity and memory," Nature Medicine, vol. 14, no. 8, pp. 837-842, 2008.

[91] E. B. Lee, L. Z. Leng, B. Zhang et al., "Targeting amyloid$\beta$ peptide $(\mathrm{A} \beta)$ oligomers by passive immunization with a conformation-selective monoclonal antibody improves learning and memory in A $\beta$ precursor protein (APP) transgenic mice," Journal of Biological Chemistry, vol. 281, no. 7, pp. 4292-4299, 2006.

[92] M. Knobloch, U. Konietzko, D. C. Krebs, and R. M. Nitsch, "Intracellular $\mathrm{A} \beta$ and cognitive deficits precede $\beta$-amyloid deposition in transgenic arcA $\beta$ mice," Neurobiology of Aging, vol. 28, no. 9, pp. 1297-1306, 2007.

[93] S. R. Hughes, S. Goyal, J. E. Sun et al., "Two-hybrid system as a model to study the interaction of $\beta$-amyloid peptide monomers," Proceedings of the National Academy of Sciences of the United States, vol. 93, no. 5, pp. 2065-2070, 1996.

[94] S. Bagriantsev and S. Liebman, "Modulation of $A \beta_{42}$ low-n oligomerization using a novel yeast reporter system," $B M C$ Biology, vol. 4, article 32, 2006.

[95] T. von der Haar, L. Jossé, P. Wright, J. Zenthon, and M. F. Tuite, "Development of a novel yeast cell-based system for studying the aggregation of Alzheimer's disease-associated A $\beta$ peptides in vivo," Neurodegenerative Diseases, vol. 4 , no. 2-3, pp. 136-147, 2007.

[96] M. F. Tuite and B. S. Cox, "The $\left[\mathrm{PSI}^{+}\right]$prion of yeast: a problem of inheritance," Methods, vol. 39, no. 1, pp. 9-22, 2006.

[97] A. D. Williams, E. Portelius, I. Kheterpal et al., "Mapping A $\beta$ amyloid fibril secondary structure using scanning proline mutagenesis," Journal of Molecular Biology, vol. 335, no. 3, pp. 833-842, 2004.

[98] S. Krobitsch and S. Lindquist, "Aggregation of huntingtin in yeast varies with the length of the polyglutamine expansion and the expression of chaperone proteins," Proceedings of the National Academy of Sciences of the United States of America, vol. 97, no. 4, pp. 1589-1594, 2000.

[99] Y. O. Chernoff, S. L. Lindquist, B. I. Ono, S. G. IngeVechtomov, and S. W. Liebman, "Role of the chaperone protein Hsp104 in propagation of the yeast prion-like factor [PSI $\left.{ }^{+}\right], "$ Science, vol. 268, no. 5212, pp. 880-884, 1995.

[100] J. Caine, S. Sankovich, H. Antony et al., "Alzheimer's A $\beta$ fused to green fluorescent protein induces growth stress and a heat shock response," FEMS Yeast Research, vol. 7, no. 8, pp. 12301236, 2007.

[101] S. Treusch, S. Hamamichi, J. L. Goodman et al., "Functional links between $\mathrm{A} \beta$ toxicity, endocytic trafficking, and Alzheimer's disease risk factors in yeast," Science, vol. 334, no. 6060, pp. 1241-1245, 2011.

[102] T. F. Outeiro and S. Lindquist, "Yeast cells provide insight into $\alpha$-synuclein biology and pathobiology," Science, vol. 302, no. 5651, pp. 1772-1775, 2003.

[103] A. D. Gitler, B. J. Bevis, J. Shorter et al., "The Parkinson's disease protein $\alpha$-synuclein disrupts cellular Rab homeostasis," Proceedings of the National Academy of Sciences of the United States of America, vol. 105, no. 1, pp. 145-150, 2008. 
[104] A. A. Cooper, A. D. Gitler, A. Cashikar et al., " $\alpha$-synuclein blocks ER-Golgi traffic and Rab1 rescues neuron loss in Parkinson's models," Science, vol. 313, no. 5785, pp. 324-328, 2006.

[105] N. M. Lewandowski, S. Ju, M. Verbitsky et al., "Polyamine pathway contributes to the pathogenesis of Parkinson disease," Proceedings of the National Academy of Sciences of the United States, vol. 107, no. 39, pp. 16970-16975, 2010.

[106] E. Yeger-Lotem, L. Riva, L. J. Su et al., "Bridging highthroughput genetic and transcriptional data reveals cellular responses to alpha-synuclein toxicity," Nature Genetics, vol. 41, no. 3, pp. 316-323, 2009.

[107] L. J. Su, P. K. Auluck, T. F. Outeiro et al., "Compounds from an unbiased chemical screen reverse both ER-toGolgi trafficking defects and mitochondrial dysfunction in Parkinson's disease models," Disease Models and Mechanisms, vol. 3, no. 3-4, pp. 194-208, 2010.

[108] Y. J. Lee, S. Wang, S. R. Slone, T. A. Yacoubian, and S. N. Witt, "Defects in very long chain fatty acid synthesis enhance $\alpha$-synuclein toxicity in a yeast model of Parkinson's disease," PLoS ONE, vol. 6, no. 1, Article ID e15946, 2011.

[109] Y. Y. Sere, M. Regnacq, J. Colas, and T. Berges, "A Saccharomyces cerevisiae strain unable to store neutral lipids is tolerant to oxidative stress induced by $\alpha$-synuclein," Free Radical Biology and Medicine, vol. 49, no. 11, pp. 1755-1764, 2010.

[110] H. G. Lee, X. Zhu, A. Nunomura, G. Perry, and M. A. Smith, "Amyloid $\beta$ : the alternate hypothesis," Current Alzheimer Research, vol. 3, no. 1, pp. 75-80, 2006.

[111] A. Kontush, C. Berndt, W. Weber et al., "Amyloid- $\beta$ is an antioxidant for lipoproteins in cerebrospinal fluid and plasma," Free Radical Biology and Medicine, vol. 30, no. 1, pp. 119-128, 2001.

[112] I. Macreadie, M. Lotfi-Miri, S. Mohotti, D. Shapira, L. Bennett, and J. Varghese, "Validation of folate in a convenient yeast assay suited for identification of inhibitors of Alzheimer's amyloid- $\beta$ aggregation," Journal of Alzheimer's Disease, vol. 15, no. 3, pp. 391-396, 2008. 


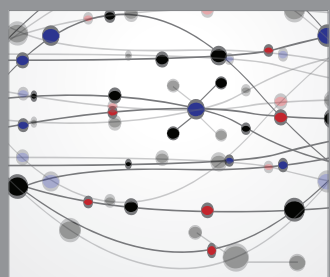

The Scientific World Journal
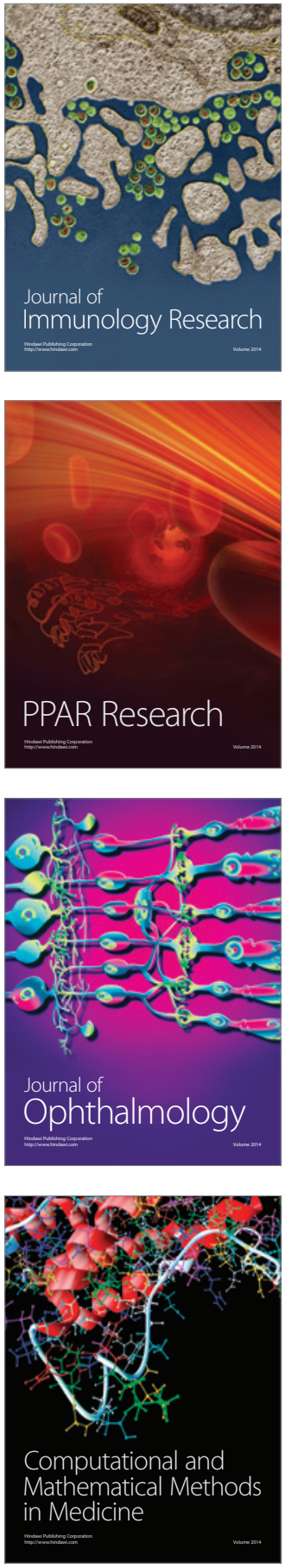

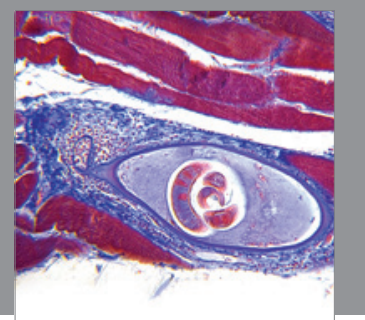

Gastroenterology

Research and Practice
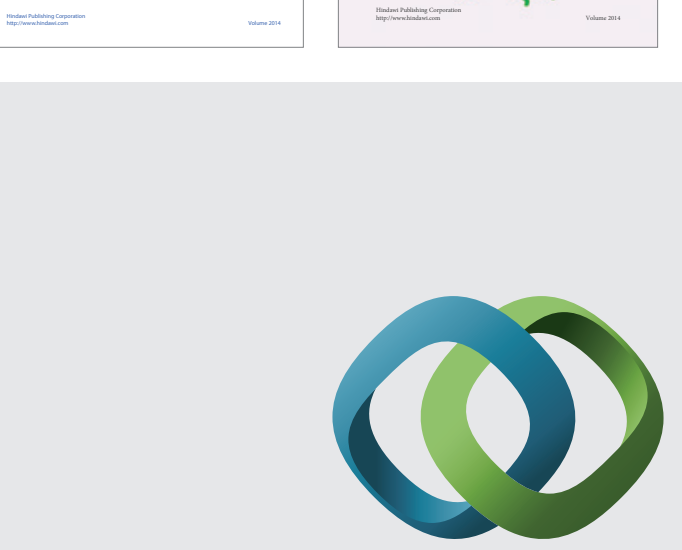

\section{Hindawi}

Submit your manuscripts at

http://www.hindawi.com
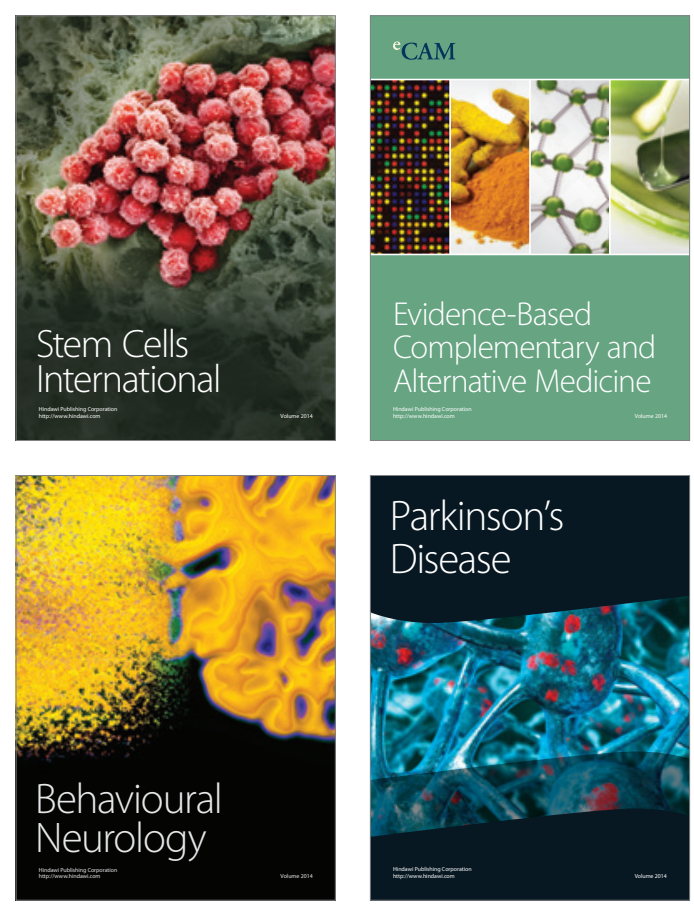

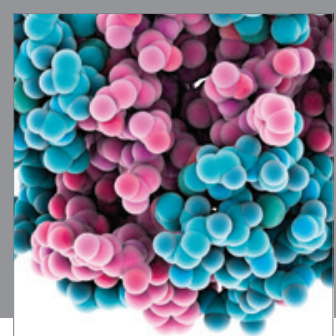

Journal of
Diabetes Research

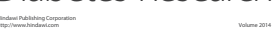

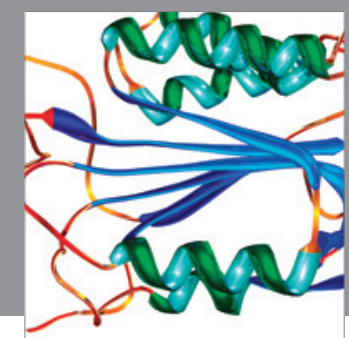

Disease Markers
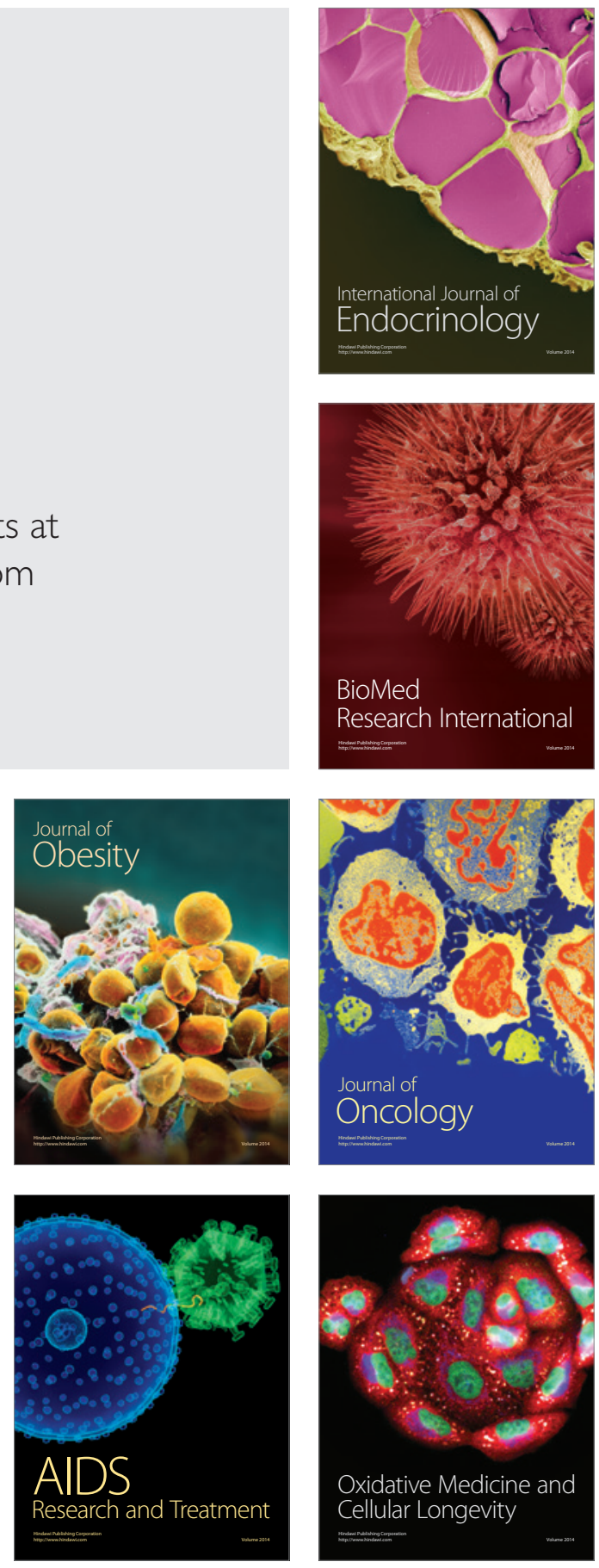